\title{
Substitutes and Stability for Matching with Contracts
}

\author{
John William Hatfield and Fuhito Kojima*
}

March 3, 2008

\begin{abstract}
We consider the matching problem with contracts of Hatfield and Milgrom (2005), and we introduce new concepts of bilateral and unilateral substitutes. We show that bilateral substitutes is a sufficient condition for the existence of a stable allocation in this framework. However, the set of stable allocations does not form a lattice under this condition, and there does not necessarily exist a doctor-optimal stable allocation. Under a slightly stronger condition, unilateral substitutes, the set of stable allocations still does not necessarily form a lattice with respect to doctors' preferences, but there does exist a doctor-optimal stable allocation, and other key results such as incentive compatibility and the rural hospitals theorem are recovered.

Journal of Economic Literature Classification Numbers: C78, D44.

Key Words: substitutes, bilateral substitutes, unilateral substitutes, matching, matching with contracts, law of aggregate demand, stability, strategy-proofness, rural hospitals theorem, group strategyproofness, lattice.
\end{abstract}

\section{Introduction}

The theory of two-sided matching markets attracts attention for its theoretical appeal and its applicability to the design of real-world institutions. The

*Hatfield: Graduate School of Business, Stanford University, Stanford, CA 94305, email: hatfield_john@gsb.stanford.edu. Kojima: Department of Economics, Harvard University, Cambridge, MA 02138, e-mail: kojima@fas.harvard.edu. We are grateful to Eric Budish, Federico Echenique, Bettina Klaus, Flip Klijn, Paul Milgrom, Michael Ostrovsky, Al Roth, Steven Tadelis, Alex Westkamp and seminar participants at Harvard and SISL Mini-Conference on Matching at Caltech for comments. 
National Resident Matching Program for matching medical residents to hospitals and the student assignment systems in New York City and Boston are examples of mechanisms designed by economists using the theory. ${ }^{1}$ Hatfield and Milgrom (2005) present a unified framework of matching with contracts, which includes the two-sided matching models and package auction models as special cases. They introduce the substitutes condition, which is one natural extension of the substitutability condition in the matching literature (Roth and Sotomayor, 1990) to matching with contracts, and show that there exists a stable allocation of contracts if contracts are substitutes. Furthermore, if contracts are substitutes, then there exists a doctor-optimal stable allocation (a stable allocation that is weakly preferred to every stable allocation by all doctors), and the set of stable allocations forms a lattice with respect to a partial order based on the doctors' common preferences.

While the substitutes condition is sufficient for many results in matching theory, it is not necessary in matching problems with contracts. Hatfield and Kojima (2007b) give an example where contracts are not substitutes but stable allocations are guaranteed to exist. We introduce a weaker condition, called bilateral substitutes, which is sufficient to guarantee the existence of a stable allocation. Contracts are bilateral substitutes for a hospital if there are no two doctors and their contracts $x, z$ and a set of contracts $Y$ with other doctors such that the hospital that regards $Y$ as available wants to $\operatorname{sign} z$ if and only if $x$ is also available. Bilateral substitutes is less restrictive than the substitutes condition of Hatfield and Milgrom (2005) and reduces to standard substitutability in matching models with no terms of contract.

While bilateral substitutes is sufficient for the existence of a stable allocation, few other results in matching theory generalize under that condition. In simple matching markets, the set of stable allocations forms a lattice and there exists a doctor-optimal stable allocation (Gale and Shapley, 1962). Neither of these properties carry over to matching with contracts if we only impose that contracts are bilateral substitutes for hospitals. Furthermore, with an additional assumption of the law of aggregate demand, in simple matching markets the same set of doctors and hospitals are matched in different stable matchings (rural hospital theorem (Roth, 1986)), the doctor-optimal stable mechanism is strategy-proof for doctors (Dubins and Freedman, 1981; Roth, 1982), and the doctor-optimal stable matching is weakly Pareto optimal for doctors, that is, there is no individually rational matching strictly preferred by every doctor (Kojima, 2007; Roth, 1982). None of these properties carry

\footnotetext{
${ }^{1}$ The theory was first developed by Gale and Shapley (1962). For applications to labor markets, see Roth (1984a) and Roth and Peranson (1999). For applications to student assignment, see for example Abdulkadiroğlu and Sönmez (2003), Abdulkadiroğlu et al. (2005a) and Abdulkadiroğlu et al. (2005b).
} 
over to matching with contracts when contracts are bilateral substitutes.

We introduce another new concept of substitutes to restore these properties. Contracts are unilateral substitutes for a hospital if there is no contract $z$ and sets of contracts $Y$ and $Y^{\prime}$ with other doctors such that $Y \subseteq Y^{\prime}$ and the hospital rejects $z$ when $Y$ is available but accepts $z$ if the larger set $Y^{\prime}$ is available. Unilateral substitutes is essential for a number of results. First, it is sufficient for the existence of a doctor-optimal stable allocation. Second, with the law of aggregate demand, it implies the rural hospitals theorem, groupstrategy-proofness of the doctor-optimal stable mechanism for the doctors, and weak Pareto optimality of the doctor-optimal stable allocation for the doctors. Even under the unilateral substitutes condition, however, there does not necessarily exist a doctor-pessimal stable allocation. As a consequence, the set of stable allocations still does not form a lattice with respect to the preferences of the doctors.

We also apply our theory to the matching problem with couples. First, we note that a matching problem with couples can be seen as a special case of matching with contracts, with an appropriate interpretation. We give an example that shows the standard substitutes condition is often violated in matching with couples. On the other hand, our results give a sufficient condition for the existence of stable matching with couples. In this sense, the generality of our analysis is not only theoretically interesting but also useful in application.

This paper highlights distinctive aspects of matching with contracts relative to more traditional matching theory. While the model of matching with contracts is more general than most existing models of matching, the often-imposed substitutes condition is analogous to conditions in the existing literature. Furthermore, the basic approach of analysis in matching with contracts has been similar to some recent works in matching theory without contracts. Beginning with Adachi (2000), the monotonic structure of matching problems has been exploited in matching problems without contracts by Echenique and Oviedo (2004, 2006) and Fleiner (2003). These works define a function that is monotone under the substitutes condition, and resort to Tarski's fixed point theorem to show the existence of fixed points, which coincide with stable matchings. ${ }^{2}$ The underlying monotonicity structure guarantees that, under substitutes, most results of matching generalize as well, such as the existence of doctor- (hospital-) optimal stable matching and (under the law of aggregate demand) the rural hospitals theorem. Our contributions

\footnotetext{
${ }^{2}$ In a more general supply chain network model, Ostrovsky (2007) introduces cross-side complementarity in addition to same-side substitutability. These conditions guarantees the monotonicity of a function, and he shows the existence of a stable allocation by Tarski's theorem.
} 
are twofold. First, we demonstrate that the monotonicity structure is not necessary to conduct meaningful analysis in matching theory since a number of results can be shown under bilateral or unilateral substitutes even though the monotonic structure is lost. Second, there is more than one relevant concept of substitutes and different properties hold under different conditions in matching with contracts, unlike more traditional matching markets without terms of contract. Our analysis suggests that matching with contracts may be a rich framework that warrants future research.

The rest of the paper proceeds as follows. Section 2 introduces the model. Section 3 introduces bilateral substitutes and show the existence of a stable allocation. Section 4 introduces unilateral substitutes and recover results that do not hold under bilateral substitutes. Section 5 concludes.

\section{The Model}

There are finite sets $D$ and $H$ of doctors and hospitals, and a finite set $X$ of contracts. Each contract $x \in X$ is associated with one doctor $x_{D} \in D$ and one hospital $x_{H} \in H$. Each doctor can sign at most one contract. The null contract, meaning that the doctor has no contract, is denoted by $\emptyset$. For each $d \in D, P_{d}$ is a strict preference relation on $\left\{x \in X \mid x_{D}=d\right\} \cup\{\emptyset\}$. A contract is acceptable if it is strictly preferred to the null contract and unacceptable if it is strictly dispreferred to the null contract. For each $d \in D$ and $X^{\prime} \subseteq X$, we define the chosen set $C_{d}\left(X^{\prime}\right)$ by

$$
C_{d}\left(X^{\prime}\right)=\max _{P_{d}}\left[\left\{x \in X^{\prime} \mid x_{D}=d\right\} \cup\{\emptyset\}\right]
$$

Let $C_{D}\left(X^{\prime}\right)=\bigcup_{d \in D} C_{d}\left(X^{\prime}\right)$ be the set of contracts chosen from $X^{\prime}$ by some doctor.

We allow each hospital to sign multiple contracts, and assume that each hospital $h \in H$ has a preference relation $P_{h}$ on the set of subsets of contracts involving it. For any $X^{\prime} \subseteq X$, define $C_{h}\left(X^{\prime}\right)$ by

$C_{h}\left(X^{\prime}\right)=\max _{P_{h}}\left\{X^{\prime \prime} \subseteq X^{\prime} \mid\left(x \in X^{\prime \prime} \Rightarrow x_{H}=h\right)\right.$ and $\left.\left(x, x^{\prime} \in X^{\prime \prime}, x \neq x^{\prime} \Rightarrow x_{D} \neq x_{D}^{\prime}\right)\right\}$.

Let $C_{H}\left(X^{\prime}\right)=\bigcup_{h \in H} C_{h}\left(X^{\prime}\right)$ be the set of contracts chosen from $X^{\prime}$ by some hospital.

We write $P_{D}=\left(P_{d}\right)_{d \in D}$ to denote a preference profile of doctors. We also write $P_{-d}$ to denote $\left(P_{d^{\prime}}\right)_{d^{\prime} \in D \backslash\{d\}}$ for $d \in D$, and $P_{D^{\prime}}$ to denote $\left(P_{d}\right)_{d \in D^{\prime}}$ and $P_{-D^{\prime}}$ to denote $\left(P_{d}\right)_{d \in D \backslash D^{\prime}}$ for $D^{\prime} \subset D$. A preference relation is extended to allocations in a natural way. For example, for two allocations $Y, Z \subseteq X$, 
we write $Y \succ_{h} Z$ to mean $\left\{y \in Y \mid y_{H}=h\right\} \succ_{h}\left\{z \in Z \mid z_{H}=h\right\}$. Similar notation will be used for doctors as long as there is no confusion. For a set of contracts $Y$, we denote $Y_{d}=\left\{y \in Y \mid y_{D}=d\right\}$ and $Y_{h}=\left\{y \in Y \mid y_{H}=h\right\}$ and denote $Y_{D}=\bigcup_{y \in Y}\left\{y_{D}\right\}$ and $Y_{H}=\bigcup_{y \in Y}\left\{y_{H}\right\}$.

A set of contracts $X^{\prime} \subseteq X$ is an allocation if $x, x^{\prime} \in X^{\prime}$ and $x \neq x^{\prime}$ imply $x_{D} \neq x_{D}^{\prime}$. That is, a set of contracts is an allocation if each doctor signs at most one contract.

Definition 1. A set of contracts $X^{\prime} \subseteq X$ is a stable allocation (or a stable set of contracts) if

1. $C_{D}\left(X^{\prime}\right)=C_{H}\left(X^{\prime}\right)=X^{\prime}$, and

2. there exists no hospital $h$ and set of contracts $X^{\prime \prime} \neq C_{h}\left(X^{\prime}\right)$ such that $X^{\prime \prime}=C_{h}\left(X^{\prime} \cup X^{\prime \prime}\right) \subseteq C_{D}\left(X^{\prime} \cup X^{\prime \prime}\right)$.

When condition (2) is violated by some $X^{\prime \prime}$, we say that $X^{\prime \prime}$ blocks $X^{\prime}$ or $X^{\prime \prime}$ is a block of $X^{\prime}$ for $h$.

We introduce two partial orders over matchings. For two allocations $Y$ and $Z$,

$$
\begin{aligned}
& Y \leq_{D} Z \Longleftrightarrow Z \succeq_{d} Y, \forall d \in D, \\
& Y \leq_{H} Z \Longleftrightarrow Z_{h}=C_{h}(Z \cup Y), \forall h \in H .
\end{aligned}
$$

In general, a nonempty set $S$ endowed with a partial order $\leq$ is said to be a lattice if

1. For any $s, s^{\prime} \in S$, there exists $s^{\prime \prime} \in S$ such that $s \leq s^{\prime \prime}, s^{\prime} \leq s^{\prime \prime}$, and $s^{\prime \prime} \leq s^{\prime \prime \prime}$ for any $s^{\prime \prime \prime}$ such that $s \leq s^{\prime \prime \prime}$ and $s^{\prime} \leq s^{\prime \prime \prime}$, and

2. For any $s, s^{\prime} \in S$, there exists $s^{\prime \prime} \in S$ such that $s^{\prime \prime} \leq s, s^{\prime \prime} \leq s^{\prime}$, and $s^{\prime \prime \prime} \leq s^{\prime \prime}$ for any $s^{\prime \prime \prime}$ such that $s^{\prime \prime \prime} \leq s$ and $s^{\prime \prime \prime} \leq s^{\prime}$.

It is easy to show that a finite lattice has a minimal element and a maximal element, that is, elements $s_{*}$ and $s^{*}$ of $S$ such that $s_{*} \leq s \leq s^{*}$ for every $s \in$ $S$. A stable allocation $Z$ is called the doctor-optimal (doctor-pessimal) stable allocation if every doctor weakly prefers (disprefers) $Z$ to every other stable allocation. Similarly, a stable allocation $Z$ is called the hospitaloptimal (hospital-pessimal) stable allocation if every hospital weakly prefers (disprefers) $Z$ to every other stable allocation. It is well-known that if the set of stable allocations is a lattice with respect to $\leq_{D}$ (respectively $\leq_{H}$ ), then there exist a doctor-optimal and doctor-pessimal stable allocations (respectively hospital-optimal and hospital-pessimal stable allocations). 


\section{Bilateral Substitutes}

The substitutability condition on hospital preferences was introduced by Kelso and Crawford (1982) in a matching model with wages and adapted widely in the matching literature with and without wages (Roth and Sotomayor, 1990). One natural extension of substitutability from matching models with a fixed contract to models with multiple contract terms is to simply let hospital preferences be substitutable over contracts instead of over doctors. This is the approach employed by Hatfield and Milgrom (2005).

Definition 2. Contracts are substitutes for $h$ if there does not exist a contract $z \in X$ and sets of contracts $Y, Y^{\prime}$ with $z \in Y \subseteq Y^{\prime}$ such that $z \notin C_{h}(Y)$ and $z \in C_{h}\left(Y^{\prime}\right)$.

In other words, contracts are substitutes if the addition of a contract to the choice set never induces a hospital to take a contract it previously rejected. Hatfield and Milgrom (2005) show that there exists a stable allocation when contracts are substitutes for every hospital.

Result 1 (Hatfield and Milgrom 2005). Suppose that contracts are substitutes for every hospital. Then the set of stable allocations forms a nonempty finite lattice with respect to $\leq_{D}$ (respectively $\leq_{H}$ ) based on the common preferences of the doctors (respectively hospitals). In particular, there exist a doctor-optimal, doctor-pessimal, hospital-optimal and hospital-pessimal stable allocations.

However, Hatfield and Kojima (2007b) show that the substitutes condition is not necessary for guaranteeing the existence of a stable allocation. ${ }^{3}$

We introduce a weakening of the substitutes condition that is sufficient to guarantee the existence of a stable allocation.

Definition 3. Contracts are bilateral substitutes for $h$ if there does not exist contracts $x, z \in X$ and a set of contracts $Y \subseteq X$ such that $x_{D}, z_{D} \notin Y_{D}$, $z \notin C_{h}(Y \cup\{z\})$ and $z \in C_{h}(Y \cup\{x, z\})$.

Bilateral substitutes is a weaker condition than substitutes in two ways. First, when we consider a rejected contract $z$, we only consider sets of other contracts that do not involve $z_{D}$. Second, when we consider a contract $x$ that may be added to the set of contracts, we only consider contracts with doctors not in $Y_{D}$. In a matching problem without contracts (i.e.,

\footnotetext{
${ }^{3}$ Theorem 5 of Hatfield and Milgrom (2005) claims that the substitutes condition is necessary for guaranteeing the existence of a stable allocation. An example of Hatfield and Kojima (2007b) implies that their result does not hold without modification.
} 
for any two contracts $x, x^{\prime} \in X, x_{D}=x_{D}^{\prime}$ and $x_{H}=x_{H}^{\prime}$ imply $x=x^{\prime}$ ), the bilateral substitutes condition coincides with the substitutes condition, and both concepts reduce to the standard substitutability condition (see for example Roth and Sotomayor (1990).)

Theorem 1. Suppose that contracts are bilateral substitutes for every hospital. Then there exists a stable allocation.

In order to prove Theorem 1, we define the following algorithm, called the doctor-proposing deferred acceptance algorithm with renegotiation.

- Step 1: One (arbitrarily chosen) doctor offers her first choice contract $x_{1}$. The hospital that is offered the contract, $h_{1}=\left(x_{1}\right)_{H}$, holds the contract if it is acceptable and rejects it otherwise. Let $A_{h_{1}}(1)=\left\{x_{1}\right\}$, and $A_{h}(1)=\emptyset$ for all $h \neq h_{1}$.

In general,

- Step $t \geq 2$ : One of the doctors for whom no contract is currently held by a hospital offers the most preferred contract, say $x_{t}$, that has not been rejected in previous steps. Let $h_{t}=\left(x_{t}\right)_{H}$ hold $C_{h_{t}}\left(A_{h}(t-1) \cup\left\{x_{t}\right\}\right)$ and reject all other contracts. Let $A_{h_{t}}(t)=A_{h_{t}}(t-1) \cup\left\{x_{t}\right\}$, and $A_{h}(t)=A_{h}(t-1)$ for all $h \neq h_{t}$.

The algorithm terminates when either every doctor is matched to a hospital or every unmatched doctor has had every acceptable contract rejected. As there are a finite number of contracts, the algorithm terminates in some finite number $T$ of steps. At that point, the algorithm produces $X^{\prime}=$ $\bigcup_{h \in H} C_{h}\left(A_{h}(T)\right)$, i.e., the set of contracts that are held by some hospital at the terminal step $T$.

The algorithm generalizes the deferred acceptance algorithm of Gale and Shapley (1962), and is equivalent to the cumulative offer process as defined by Hatfield and Milgrom (2005). In this algorithm, a hospital $h$ has accumulated offers in the set of contracts $A_{h}(t)$ by time $t$, and $h$ always chooses the best set of offers from it. Without assumptions on hospital preferences, the algorithm does not guarantee feasibility of the resulting set of contracts, that is, each doctor has at most one contract. The first part of the proof of Theorem 1 shows that the algorithm produces a feasible allocation when contracts are bilateral substitutes.

Proof of Theorem 1. Consider the doctor-proposing deferred acceptance algorithm with renegotiation. Suppose that contracts are bilateral substitutes for every hospital. We first show that for every $h \in H$, $z$ with $z_{H}=h$ 
and $t \geq 2$, if $z \in A_{h}(t-1)$ and $z_{D} \notin\left[C_{h}\left(A_{h}(t-1)\right)\right]_{D}$ then $z \notin C_{h}\left(A_{h}(t)\right)$. This implication is obvious if no contract is offered to $h$ in step $t$ since $A_{h}(t)=A_{h}(t-1)$. Also it is obvious if $z_{D}$ offers a contract to $h$ at step $t$. Thus suppose that a contract $x_{t}$ is offered to $h$ at step $t$, where $\left(x_{t}\right)_{D} \neq z_{D}$. Let $Y=A_{h}(t-1) \backslash\left\{y \in X \mid y_{D} \in\left\{\left(x_{t}\right)_{D}, z_{D}\right\}\right\}$. By definition, $\left(x_{t}\right)_{D} \notin Y_{D}$ and $z_{D} \notin Y_{D}$. Since $\left(x_{t}\right)_{D}$ is making an offer at step $t,\left(x_{t}\right)_{D} \notin\left[C_{h}\left(A_{h}(t-1)\right)\right]_{D}$. Also, by assumption $z_{D} \notin\left[C_{h}\left(A_{h}(t-1)\right)\right]_{D}$. Therefore $z \notin C_{h}\left(A_{h}(t-1)\right)=$ $C_{h}(Y \cup z)$. By bilateral substitutes, $z \notin C_{h}\left(Y \cup\{z\} \cup\left\{x_{t}\right\}\right)$ and hence $z \notin C_{h}\left(A_{h}(t)\right) .{ }^{4}$ This observation shows that the algorithm produces a (feasible) allocation.

To prove the theorem, consider the allocation $X^{\prime}$ generated by the doctorproposing deferred acceptance algorithm with renegotiation (and let $T$ be the step in which the algorithm terminated). We have $C_{D}\left(X^{\prime}\right)=C_{H}\left(X^{\prime}\right)=X^{\prime}$ by definition of $X^{\prime}$, so the first condition for stability is satisfied. To prove the second condition for stability suppose, for contradiction, that there exists a hospital $h$ and a set of contracts $X^{\prime \prime} \neq C_{h}\left(X^{\prime}\right)$ such that $X^{\prime \prime}=C_{h}\left(X^{\prime} \cup X^{\prime \prime}\right) \subseteq$ $C_{D}\left(X^{\prime} \cup X^{\prime \prime}\right)$. The condition $X^{\prime \prime} \subseteq C_{D}\left(X^{\prime} \cup X^{\prime \prime}\right)$ implies that $x^{\prime \prime} \succeq_{x_{D}^{\prime \prime}} X^{\prime}$ for all $x^{\prime \prime} \in X^{\prime \prime}$. Also note that $X_{H}^{\prime \prime}=\{h\}$ since $X^{\prime \prime}=C_{h}\left(X^{\prime} \cup X^{\prime \prime}\right)$. These two observations and the definition of the algorithm imply that $X^{\prime \prime} \subseteq A_{h}(T)$, and hence $X^{\prime \prime}=C_{h}\left(X^{\prime} \cup X^{\prime \prime}\right)=C_{h}\left(A_{h}(T) \cup X^{\prime \prime}\right)=C_{h}\left(A_{h}(T)\right)=C_{h}\left(X^{\prime}\right)$. This equality contradicts the assumption $X^{\prime \prime} \neq C_{h}\left(X^{\prime}\right)$, completing the proof.

Theorem 1 shows that bilateral substitutes is sufficient for the existence of a stable allocation. Note that it is necessary to use the doctor-proposing deferred acceptance algorithm with renegotiation in order to find a stable allocation: use of the doctor-proposing algorithm (without renegotiation) when preferences satisfy bilateral substitutes but not substitutes will not necessarily result in a stable allocation. Consider the following example:

$$
\begin{aligned}
P_{h}:\{x, z\} \succ_{h}\{\tilde{z}\} \succ_{h}\{\tilde{x}\} \succ_{h}\{x\} \succ_{h}\{z\}, \quad & P_{x_{D}}: \quad \tilde{x} \succ_{x_{D}} x, \\
& P_{z_{D}}: \quad z \succ_{z_{D}} \tilde{z} .
\end{aligned}
$$

Under the standard doctor-proposing algorithm, when $x_{D}$ proposes his favorite contract $\tilde{x}, z_{D}$ must propose $\tilde{z}$ in order to be hired at hospital $h$. However, at that point, $x_{D}$ will propose $x$. Since hospital $h$ has access to only $\tilde{z}$ under the standard algorithm, he will reject $x$, and the final allocation will be $\{\tilde{z}\}$, which is unstable, as all three agents agree that $\{x, z\}$ is a

\footnotetext{
${ }^{4}$ This last implication is shown by contraposition as follows. Suppose $z \in C_{h}\left(A_{h}(t)\right)$. Since $z \notin C_{h}\left(A_{h}(t-1)\right)$ and $A_{h}(t)=A_{h}(t-1) \cup\left\{x_{t}\right\}$ by assumption, this implies $x_{t} \in$ $C_{h}\left(A_{h}(t)\right)$. Since, for each doctor, at most one contract with her is in $C_{h}\left(A_{h}(t)\right)$, by $z, x_{t} \in C_{h}\left(A_{h}(t)\right)$ we obtain $z \in C_{h}\left(Y \cup\{z\} \cup\left\{x_{t}\right\}\right)$.
} 
better allocation. The doctor-proposing algorithm with renegotiation will result in the stable allocation $\{x, z\}$, as when $x_{D}$ proposes $x$, hospital $h$ will renegotiate with $z_{D}$ for the contract $z$. This is in a contrast with the cumulative offer process when contracts are subsitutes, as studied by Hatfield and Milgrom (2005). When contracts are substitutes, the hospital never wishes to renegotiate. We further study this issue in Section 4, and show that a more general condition guarantees that there is no renegotiation during the algorithm.

Theorem 1 generalizes previous results in the matching literature. In particular, the result subsumes the existence result in Hatfield and Milgrom (2005) who show that there exists a stable allocation under the stronger assumption that contracts are substitutes for all hospitals. The above proof is also different from theirs. In Hatfield and Milgrom (2005), the substitutes condition guarantees the monotonicity of the doctor-proposing algorithm, and renegotiation is not needed, while it is necessary when only bilateral substitutes is required.

\subsection{Insufficiency of Bilateral Substitutes for Additional Results}

While bilateral substitutes is sufficient for the existence of a stable allocation in the matching with contracts framework, it does not impose enough structure on the preferences of the hospitals to obtain other key results in the matching literature. For instance, the bilateral substitutes condition does not guarantee the existence of a doctor-optimal stable allocation. Consider the following example:

$$
\begin{array}{lll}
P_{h}:\{x, z\} \succ_{h}\{\tilde{z}\} \succ_{h}\{\tilde{x}\} \succ_{h}\{x\} \succ_{h}\{z\}, & P_{x_{D}}: \quad \tilde{x} \succ_{x_{D}} x, \\
& P_{z_{D}}:\left\{z_{h^{\prime}}:\left\{\succ_{z_{D}} z^{\prime} \succ_{z_{D}} \tilde{z} .\right.\right.
\end{array}
$$

There are two stable allocations: $\left\{\tilde{x}, z^{\prime}\right\}$ and $\{x, z\}$. However, $x_{D}$ prefers the former and $z_{D}$ prefers the latter, and so there does not exist either a doctor-optimal or a doctor-pessimal stable allocation. As a consequence, the set of stable allocations does not form a lattice with respect to a partial order $\leq_{D}$ based on preferences of doctors: this is in contrast to the case with the substitutes condition, in which the set of stable allocations forms a lattice with respect to $\leq_{D}$ (Roth, 1985; Hatfield and Milgrom, 2005). Similarly, $h$ prefers $\{x, z\}$ while $h^{\prime}$ prefers $\left\{\tilde{x}, z^{\prime}\right\}$, so there is neither a hospital-optimal nor hospital-pessimal stable allocation.

Other results in the matching literature also fail under the bilateral substitutes condition. For example, although the preferences of both hospitals 
satisfy the law of aggregate demand (Definition 6) in the above example, the rural hospitals theorem (as formalized in Theorem 5) fails: $h^{\prime}$ obtains a doctor in the former allocation but does not do so in the latter. Finally, note that no mechanism that chooses stable allocations can be strategy-proof for the doctors. If the mechanism chooses the stable allocation $\{x, z\}$ when preferences of $x_{D}$ and $z_{D}$ are $P_{x_{D}}$ and $P_{z_{D}}$ respectively, $x_{D}$ can profitably misrepresent his preferences as $P_{x_{D}}^{\prime}: \tilde{x}$, in which case the only stable allocation is $\left\{\tilde{x}, z^{\prime}\right\}$. On the other hand, if the mechanism chooses $\left\{\tilde{x}, z^{\prime}\right\}$ when preferences of $x_{D}$ and $z_{D}$ are $P_{x_{D}}$ and $P_{z_{D}}$ respectively, then $z_{D}$ can profitably misrepresent his preferences as $P_{z_{D}}^{\prime}: z \succ_{z_{D}}^{\prime} \tilde{z}$, so that the only stable allocation is $\{x, z\}$. These results are in contrast to existing results, in particular to Hatfield and Milgrom (2005), who show the above version of the rural hospitals theorem and strategy-proofness result hold under substitutes and the law of aggregate demand. Interestingly, the optimal strategic deviation for $z_{D}$ is not to simply truncate his preference list. This observation is in contrast to matching problems without contracts in which, for any stable mechanism, an optimal deviation by a doctor can always be made in the form of simply truncating the list of acceptable matches (Roth and Vande Vate, 1991). ${ }^{5}$ On the other hand, the strategy in the above example is within the class of dropping strategies as defined by Kojima and Pathak (2007). ${ }^{6}$

\subsection{Application: Stable Matching with Couples}

Our results give a sufficient condition for the existence of a stable allocation in a general model of matching with contracts. In this section, we consider the application of our results to problems of matching with couples.

In a matching problem with couples, $H$ and $C$ are the sets of hospitals and couples of doctors, respectively. ${ }^{7}$ Each couple $c=\left(m_{c}, f_{c}\right)$ is composed of two members, $m_{c}$ and $f_{c}$. Couples have preferences over pairs of hospitals and being unemployed. Each hospital has one position to fill and has preferences over doctors and being unmatched. Stable matching is defined in the usual way.

\footnotetext{
${ }^{5}$ Also in matching markets with incomplete information, Roth and Rothblum (1999) show that a best response of an agent takes the form of truncation strategy under symmetric information.

${ }^{6}$ The class of truncation strategies may not be exhaustive for hospitals. Kojima and Pathak (2007) show that a wider class called dropping strategies exhausts the optimal manipulations in many-to-one matching without contracts when hospitals have responsive preferences.

${ }^{7}$ We do not explicitly model doctors who are single. This is without loss of generality, since a single doctor can be modeled as a couple who always wants one fixed member to be unemployed.
} 
A matching problem with couples can be seen as a special instance of a matching problem with contracts as follows. Each couple can sign at most two contracts (one for each member), and each hospital can sign one contract (a couple in a couple problem plays the role of a hospital in our contract setting, and a hospital in a couple problem plays the role of a doctor in our contract setting $).{ }^{8}$ For any couple $c=\left(m_{c}, f_{c}\right)$ and hospital $h$, there are two possible contracts between $c$ and $h$ : one of them is a contract that prescribes "to match $m_{c}$ of couple $c$ to hospital $h$ " and the other is a contract "to match $f_{c}$ to $h . "$

With the above interpretation, a matching problem with couples can be seen as a special case of the matching problem with contracts. Therefore Theorems 1 and implies that bilateral substitutes (of couples' preferences) is a sufficient condition for the existence of a stable matching with couples.

Interestingly, the substitutes condition of Hatfield and Milgrom (2005) is rarely satisfied in matching problems with couples. A couple's preferences are responsive if when the hospital matched to one member of a couple improves according to that member's individual preferences, then the pair of hospitals matched to the couple as a whole improves according to the couple's preferences. ${ }^{9}$ Theorem 3.3 of Klaus and Klijn (2005) (as corrected by Klaus et al. (2006)) shows that a stable matching exists if preferences of couples satisfy weak responsiveness (weak responsiveness is a slightly weaker condition than responsiveness). Moreover, their Theorem 3.5 shows that weak responsiveness is necessary for guaranteeing the existence of a stable matching under an additional condition called restricted strict unemployment aversion, which requires that, for any pair of acceptable positions for the couple, the couple is made worse off if one of its members loses his or her acceptable position.

Theorem 1 sheds these results in a new light. Hatfield and Kojima (2007b) point out that the responsive preferences of Klaus and Klijn (2005) and Klaus et al. (2006) may violate the substitutes condition, so a matching problem with couples is an important class for which previous results of Hatfield and

\footnotetext{
${ }^{8}$ Our assumption that each hospital has only one position is important for the isomorphism presented here. If hospitals have more than one position, the corresponding problem would be a many-to-many matching problem with contracts, which is beyond the scope of the current paper (for many-to-many matching and its generalization, see for example Konishi and Ünver (2006), Echenique and Oviedo (2006) and Ostrovsky (2007)). While restrictive, the assumption of a single position for hospitals is a standard assumption in the literature, for example see Klaus and Klijn (2005).

${ }^{9}$ Responsiveness as defined by Klaus and Klijn (2005) is different from the standard one by Roth (1985). Contracts are substitutes if preferences are responsive in the sense of Roth (1985), but they may not be substitutes even if preferences are responsive in the sense of Klaus and Klijn (2005).
} 
Milgrom (2005) are not applicable. Theorem 1 gives a sufficient condition for the existence result with couples.

Example 1. [Based on Hatfield and Kojima (2007b)] In this example, a couple has preferences that violate the substitutes condition while satisfying the bilateral substitutes condition. There are two hospitals $h$ and $h^{\prime}$, and preference relation $P_{c}$ of couple $c=\left(m_{c}, f_{c}\right)$ is given by:

$$
P_{c}:\left\{\left(h^{\prime}, m_{c}\right),\left(h, f_{c}\right)\right\} \succ_{c}\left\{\left(h, m_{c}\right)\right\} \succ_{c}\left\{\left(h^{\prime}, m_{c}\right)\right\} \succ_{c}\left\{\left(h, f_{c}\right)\right\},
$$

where $\left(\tilde{h}, i_{c}\right)$ denotes a contract that matches hospital $\tilde{h} \in\left\{h, h^{\prime}\right\}$ to a member $i_{c} \in\left\{m_{c}, f_{c}\right\}$ of couple $c$. This preference relation of $c$ violates the substitutes condition since $\left(h^{\prime}, m_{c}\right) \notin C_{c}\left(\left\{\left(h^{\prime}, m_{c}\right),\left(h, m_{c}\right)\right\}\right)$ but we have $\left(h^{\prime}, m_{c}\right) \in C_{c}\left(\left\{\left(h^{\prime}, m_{c}\right),\left(h, m_{c}\right),\left(h, f_{c}\right)\right\}\right)$. By contrast, the preferences can be shown to satisfy the bilateral substitutes condition. Therefore there exists a stable allocation if preferences of other couples also satisfy the bilateral substitutes condition.

The above preference $P_{c}$ may be natural in some situations. Suppose, for example, $h$ is a position with high wage and long working hours, while $h^{\prime}$ is a position with low wage and short working hours. If the female member $f_{c}$ is unemployed, the couple prefers for the male member $m_{c}$ to work at $h$ rather than $h^{\prime}$ to earn high wages. It is better for the couple, however, for the male member to work at $h^{\prime}$ with short work hours if the female member also works at $h$.

Example 2. In this example, a couple has preferences that violate the (weak) responsiveness condition (Klaus and Klijn, 2005; Klaus et al., 2006) while satisfying the bilateral substitutes condition. There are two hospitals $h$ and $h^{\prime}$, and a preference relation $P_{c}$ of couple $c=\left(m_{c}, f_{c}\right)$ given by (with notation analogous to Example 1):

$$
P_{c}:\left\{\left(h, m_{c}\right)\right\} \succ_{c}\left\{\left(h^{\prime}, f_{c}\right)\right\} .
$$

These preferences violate the (weak) responsiveness condition, since the couple should prefer $\left\{\left(h, m_{c}\right),\left(h^{\prime}, f_{c}\right)\right\}$ to $\left\{\left(h, m_{c}\right)\right\}$ and $\left\{\left(h^{\prime}, f_{c}\right)\right\}$ if they had responsive preference and $\left\{\left(h, m_{c}\right)\right\}$ and $\left\{\left(h^{\prime}, f_{c}\right)\right\}$ are acceptable. This preference relation satisfies the bilateral substitutes condition, so there exists a stable allocation if preferences of other couples also satisfy the bilateral substitutes condition.

The above preference $P_{c}$ has an interpretation that may be natural in some labor markets such as those for medical doctors and hospitals. Hospitals $h$ and $h^{\prime}$ are located in different cities. The "unemployement" option is 
interpreted as an outside option, for example consultant jobs. ${ }^{10}$ The couple wants to live together, so it is unacceptable for one member to work in $h$ and the other to work in $h^{\prime}$. Thus the couple's only acceptable choices are for one member of the couple to work at a hospital and the other to work in a consulting firm in the same city.

\section{Unilateral Substitutes}

We have seen that bilateral substitutes is a useful notion in matching with contracts in the sense that it is the weakest condition guaranteeing the existence of a stable allocation known to date. However, as we have shown, other key results in matching theory do not hold even if contracts are bilateral substitutes. Thus we consider a strengthening of the bilateral substitutes condition.

Definition 4. Contracts are unilateral substitutes for $h$ if there does not exist $z \in X$ and subsets of contracts $Y, Y^{\prime} \subseteq X$ with $Y \subseteq Y^{\prime}$ such that $z_{D} \notin Y_{D}, z \notin C_{h}(Y \cup\{z\})$ and $z \in C_{h}\left(Y^{\prime} \cup\{z\}\right) .{ }^{11}$

It is clear by definition that the substitutes condition implies the unilateral substitutes condition, and that the unilateral substitutes condition implies the bilateral substitutes condition. All of these conditions coincide in matching problems without contracts. To investigate further relationships between these conditions, we introduce the following property, somewhat similar to Pareto separability of Roth (1984b).

Definition 5. Preferences of $h$ are Pareto separable if, for any $x, x^{\prime}$ with $x_{D}=x_{D}^{\prime}$ and $x_{H}=x_{H}^{\prime}=h$, if $x \in C_{h}\left(Y \cup\left\{x, x^{\prime}\right\}\right)$ for some $Y \subset X$, then $x^{\prime} \notin C_{h}\left(Y^{\prime} \cup\left\{x, x^{\prime}\right\}\right)$ for any $Y^{\prime} \subset X$.

Preferences are Pareto separable for a hospital if the hospital's choice between $x$ and $x^{\prime}$, two contracts with the same doctor, do not depend on what other contracts the hospital has access to.

\footnotetext{
${ }^{10}$ The unemployement option for a doctor in our model does not need to be taken literally as unemployment. Many applications of matching theory focus on a particular centralized matching market such as the National Resident Matching Market, and jobs outside the particular market can be treated as unemployment. Note that, in application, many doctors may become "unemployed" in this sense; for example, consulting companies regularly hire medical doctors.

${ }^{11}$ Note that unilateral substitutes condition is equivalent to the condition that there does not exist contracts $x, z \in X$ and a set of contracts $Y \subseteq X$ such that $z_{D} \notin Y_{D}$, $z \notin C_{h}(Y \cup\{z\})$ and $z \in C_{h}(Y \cup\{x, z\})$. This property will be used in several ensuing proofs.
} 
Theorem 2. Preferences of a hospital satisfy substitutes if and only if they satisfy unilateral substitutes and Pareto separability.

Proof. The "if" part. Suppose $x$ is not in $C_{h}(Y \cup\{x\})$ and consider adding a contract $z$. If $x_{D}$ is not in $Y_{D}$, then unilateral substitutes implies that $x \notin C_{h}(Y \cup\{x, z\})$. So suppose $x_{D} \in Y_{D}$. If $x_{D}$ is not in $\left[C_{h}(Y \cup\{x\})\right]_{D}$, then we can take a subset $Y^{\prime}$ of $Y$ such that $x_{D}$ is not in $Y_{D}^{\prime}$ and $x \notin$ $C_{h}\left(Y^{\prime} \cup\{x\}\right)$, and unilateral substitutes implies that $x \notin C_{h}(Y \cup\{x, z\})$. If $x^{\prime}$ is in $C_{h}(Y \cup\{x\})$ for some $x^{\prime}$ with $x_{D}^{\prime}=x_{D}$, then by Pareto separability, $x \notin C_{h}(Y \cup\{x, z\})$, which shows that the substitutes condition is satisfied.

The "only if" part. It is obvious that the substitutes condition implies the unilateral substitutes condition. To prove that the substitutes condition implies Pareto separability, suppose $x \in C_{h}\left(Y \cup\left\{x, x^{\prime}\right\}\right)$ for some $Y$ and $x, x^{\prime} \in X$ with $x_{D}=x_{D}^{\prime}$. Then $x \in C_{h}\left(\left\{x, x^{\prime}\right\}\right)$ by the substitutes condition. Since $x_{D}$ can sign only one contract, $x^{\prime} \notin C_{h}\left(\left\{x, x^{\prime}\right\}\right)$. Since contracts are substitutes for $h$, this implies that $x^{\prime} \notin C_{h}\left(Y^{\prime} \cup\left\{x, x^{\prime}\right\}\right)$ for any $Y^{\prime} \subseteq X$.

The unilateral substitutes condition in Theorem 2 cannot be replaced with the bilateral substitutes condition, since the "if" direction cannot be strengthened to the case with bilateral substitutes. ${ }^{12}$ For example, the preference relation of a hospital $h$ given by $P_{h}:\{x, z\} \succ_{h}\{z\} \succ_{h}\left\{z^{\prime}\right\} \succ_{h}\{x\}$, with $z_{D}=z_{D}^{\prime} \neq x_{D}$ satisfies bilateral substitutes and Pareto separability but violates the substitutes condition, as $x \in C_{h}\left(\left\{x, z, z^{\prime}\right\}\right)$ but $x \notin C_{h}\left(\left\{x, z^{\prime}\right\}\right)$.

If contracts are unilateral substitutes, then the "renegotiation" does not happen in the doctor-proposing deferred acceptance algorithm with renegotiation. More formally,

Theorem 3. Suppose that $z$ is held by hospital h at a step $t$ of the doctorproposing deferred acceptance algorithm with renegotiation. At any later step $t^{\prime}>t$, if $z_{D}$ has not been rejected by $h$ since $t$, then $z$ is still held by $h$.

Proof. Suppose the contrary. Consider the first step, say step $t^{\prime}$, that some hospital $h$ wishes to obtain a set of contracts $\{z, w, \ldots, v\}$ that the hospital has previously rejected, and say this happens when he receives an offer $x$. Hence if the hospital just before receiving $x$ is currently holding a set of contracts $Y$, then no elements of $\{z, w, \ldots, v\}$ are in $Y=C_{h}(Y \cup$ $\{z, w, \ldots, v\})$. If there is a contract $y \in\{z, w, \ldots, v\}$ with $y_{D} \notin Y_{D}$, then since $y$ is in $C_{h}(\{x\} \cup Y \cup\{z, w, \ldots, v\})$ we have a violation of unilateral substitutes. If $\{z, w, \ldots, v\}_{D}$ is a subset of $Y_{D}$, denote the contracts with $\left\{z_{D}, w_{D}, \ldots, v_{D}\right\}$ in $Y$ by $\left\{z^{\prime}, w^{\prime}, \ldots, v^{\prime}\right\}$. Note that $P_{y_{D}}: y \succ_{y_{D}} y^{\prime}$ for all

\footnotetext{
${ }^{12}$ It is straightforward that the "only if" direction holds for bilateral substitutes.
} 
$y_{D} \in\left\{z_{D}, w_{D}, \ldots, v_{D}\right\}$ since hospital $h$ is holding $y^{\prime}$ at the beginning of step $t^{\prime}$ and rejected $y$ before step $t^{\prime}$. Let the set of contracts that hospital $h$ held immediately after rejecting $z$ be $Y^{\prime}$ and note that $z_{D}$ is not in $Y_{D}^{\prime}$. Then $z$ is not in $C_{h}\left(Y^{\prime} \cup\{z\}\right)$ but is in $C_{h}\left(\{x\} \cup Y^{\prime \prime} \cup\{z\}\right)$, where $Y^{\prime \prime}$ is the set of all contracts doctors offered to $h$ by step $t^{\prime}$. Since $Y^{\prime} \subseteq Y^{\prime \prime}$, these relations contradict the assumption of unilateral substitutes.

When no renegotiation occurs in the doctor-proposing deferred acceptance algorithm with renegotiation as in Theorem 3, we simply refer to the algorithm as the doctor-proposing deferred acceptance algorithm.

Theorem 4. Suppose that contracts are unilateral substitutes for every hospital. Then there exists a doctor-optimal stable allocation. The allocation that is produced by the doctor-proposing deferred acceptance algorithm is the doctor-optimal stable allocation.

Proof. To show the claim, it suffices to show that no contract $z$ that is an element of some stable allocation is ever rejected during the execution of the doctor-proposing deferred acceptance algorithm. To obtain a contradiction, suppose the contrary. Consider the first step in the algorithm at which a hospital $h$ rejects a contract $z$ that is an element of some stable allocation. Then $z \notin Y=C_{h}(Y \cup\{z\})$ where $Y$ is the set of contracts held at this step. Since $z$ is rejected at this step, $Y$ does not include any contract with $z_{D}$. By assumption, there exists some stable allocation $X^{\prime}$ with $z \in X^{\prime}$. Furthermore, every other doctor in $Y_{D}$ is weakly worse off under $X^{\prime}$ than under $Y$ since we are considering the first time in the algorithm at which $h$ rejects a contract in some stable allocation. Consider the choice of hospital $h$ from $Y \cup X^{\prime}$. If $h$ chooses any set that does not include $z$, then the allocation $X^{\prime}$ is unstable, a contradiction. If $h$ chooses some set that includes $z$, then we have violated unilateral substitutes, since $z \in C_{h}\left(X^{\prime} \cup Y\right)$ but $z \notin C_{h}(Y \cup\{z\})$ even though $z \in X^{\prime}$ and $z_{D} \notin Y_{D}$.

Theorem 4 extends previous results in matching theory. In matching without contracts, the substitutability condition is sufficient both for the existence of a stable allocation and the existence of a doctor-optimal stable allocation. A notable point of the current theorem is that, in the matching problem with contracts, different conditions are needed for these results: bilateral substitutes for the existence of a stable allocation and unilateral substitutes for the existence of a doctor-optimal stable allocation.

To study welfare for hospitals, we first extend a classical "opposition of interests" property (Theorem 9 of Roth (1985)) to our setting. ${ }^{13}$

\footnotetext{
${ }^{13}$ Roth (1985) obtain the conclusion assuming that hospital preferences satisfy the sub-
} 
Lemma 1. If $Y$ and $Z$ are two stable allocations and $Y \succeq_{d} Z$ for all $d \in D$, then $Z \succeq_{h} Y$ for all $h$.

Proof. Suppose, for contradiction, that $Y \succ_{h} Z$ for some $h \in H$. By assumption, $Y \succeq_{d} Z$ for all $d \in\left[C_{h}(Y \cup Z)\right]_{D}$. Moreover, $C_{h}(Y \cup Z) \succeq_{h} Y \succ_{h}$ $Z$ by assumption. Thus $C_{h}(Y \cup Z)$ blocks $Z$, which contradicts stability of $Z$.

The following result is an immediate corollary of the above claim and Theorem 4.

Corollary 1. Suppose that contracts are unilateral substitutes for every hospital. Then there exists a hospital-pessimal stable allocation. The doctoroptimal stable allocation and the hospital-pessimal stable allocation coincide.

While Theorem 4 shows that there exists a doctor-optimal stable allocation under unilateral substitutes, the set of allocations still do not satisfy a lattice structure with respect to doctors' common preferences. Indeed, there may not even be a doctor-pessimal stable allocation. Consider the following preferences:

$$
\begin{aligned}
P_{h}: & \left\{x, y^{\prime \prime}\right\} \succ_{h}\left\{x^{\prime \prime}, y\right\} \succ_{h}\left\{x^{\prime}, y^{\prime}\right\} \succ_{h} & P_{x_{D}}: & x^{\prime} \succ_{x_{D}} x \succ_{x_{D}} x^{\prime \prime} \\
& \left\{x^{\prime \prime}, y^{\prime \prime}\right\} \succ_{h}\left\{x^{\prime \prime}, y^{\prime}\right\} \succ_{h}\left\{x^{\prime}, y^{\prime \prime}\right\} \succ_{h} & P_{y_{D}}: & y^{\prime} \succ_{y_{D}} y \succ_{y_{D}} y^{\prime \prime} \\
& \left\{x^{\prime}, y\right\} \succ_{h}\left\{x, y^{\prime}\right\} \succ_{h}\{x, y\} \succ_{h} & & \\
& \left\{x^{\prime \prime}\right\} \succ_{h}\left\{y^{\prime \prime}\right\} \succ_{h}\left\{x^{\prime}\right\} \succ_{h}\left\{y^{\prime}\right\} \succ_{h}\{x\} \succ_{h}\{y\} & &
\end{aligned}
$$

The preferences of hospital $h$ satisfy unilateral substitutes but not substitutes. There are three stable allocations: the doctor-optimal stable allocation $\left\{x^{\prime}, y^{\prime}\right\}$ and two other stable allocations, $\left\{x^{\prime \prime}, y\right\},\left\{x, y^{\prime \prime}\right\}$. There exists no doctor-pessimal stable allocation, since $\left\{x^{\prime}, y^{\prime}\right\} \succ_{x_{D}}\left\{x, y^{\prime \prime}\right\} \succ_{x_{D}}\left\{x^{\prime \prime}, y\right\}$ while $\left\{x^{\prime}, y^{\prime}\right\} \succ_{y_{D}}\left\{x^{\prime \prime}, y\right\} \succ_{y_{D}}\left\{x, y^{\prime \prime}\right\}$. Since the existence of a doctorpessimal stable allocation is necessary for the lattice structure with respect to doctors' preferences, the lattice structure does not exist.

\subsection{The Rural Hospitals Theorem, Incentive Compat- ibility and Welfare}

We now consider the implications of unilateral substitutes when coupled with the law of aggregate demand, introduced by Hatfield and Milgrom (2005). ${ }^{14}$

Definition 6. The preferences of hospital $h \in H$ satisfy the law of aggregate demand if for all $X^{\prime} \subseteq X^{\prime \prime} \subseteq X,\left|C_{h}\left(X^{\prime}\right)\right| \leq\left|C_{h}\left(X^{\prime \prime}\right)\right|$.

stitutes condition. The current Lemma 1 extends the property to general preferences.

${ }^{14}$ Analogous conditions called cardinal monotonicity and size monotonicity are introduced by Alkan (2002) and Alkan and Gale (2003). 
Using this condition, we first show a version of the result known in the literature as the "rural hospitals theorem." Roth (1986) shows the rural hospitals theorem for many-to-one matching with responsive preferences. More specifically, he shows that every hospital that has unfilled positions at some stable match is assigned exactly the same doctors at every stable match. Martinez et al. (2000) generalize the theorem for substitutable and $q$-separable preferences. Although there is no obvious notion of "unfilled positions" under the unilateral substitutes condition and the law of aggregate demand ${ }^{15}$, Theorem 5 below shows that a version of the rural hospitals theorem still holds. More specifically, every hospital signs exactly the same number of contracts at every stable allocation, although the doctors assigned and the terms of contract can vary.

Theorem 5. If hospital preferences satisfy unilateral substitutes and the law of aggregate demand, then every doctor and hospital signs the same number of contracts at every stable allocation.

Proof. By Theorem 4, there exists a doctor-optimal stable allocation $\bar{X}$. Consider any stable allocation $X^{\prime}$. Let $A\left(X^{\prime}\right)=\left\{x \in X \mid x \succeq_{x_{D}} C_{x_{D}}\left(X^{\prime}\right)\right\}$. Since $\bar{X}$ is a doctor-optimal stable allocation, we have $A(\bar{X}) \subseteq A\left(X^{\prime}\right)$. By the law of aggregate demand, every hospital accepts at least as many contracts (and hence at least as many doctors) at $X^{\prime}=C_{H}\left(A\left(X^{\prime}\right)\right)$ as at $\bar{X}=C_{H}(\bar{X})$. On the other hand, every doctor who is matched at $X^{\prime}$ is matched at $\bar{X}$ since $X^{\prime}$ is stable and hence individually rational and $\bar{X}$ is weakly preferred by every doctor to $X^{\prime}$. Therefore every hospital and doctor signs exactly the same number of contracts.

Theorem 5 is applicable to the original domains of Roth (1986) and Martinez et al. (2000). Hatfield and Milgrom (2005) show the theorem for substitutes and the law of aggregate demand. They further show that the conclusion of the rural hospitals theorem fail if hospital preferences violate the law of aggregate demand. Theorem 5 improves upon Hatfield and Milgrom

\footnotetext{
${ }^{15}$ The most obvious extension of the number of positions at hospital $h$ is the number of contracts signed by $h$ when every contract involving $h$ is available to $h$. Unfortunately, using this definition of the number of positions, a stronger version of the rural hospitals theorem does not hold, even when preferences satisfy substitutes and the law of aggregate demand. Consider the following example:

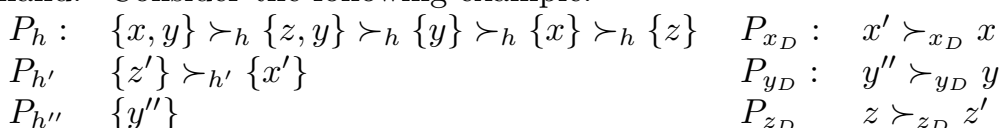

There are two stable allocations: $\left\{x^{\prime}, y^{\prime \prime}, z\right\}$ and $\left\{x, y^{\prime \prime}, z^{\prime}\right\}$. However, even though $h$ does have an unfilled position for both allocations, he nevertheless recieves different doctors under the two stable allocations.
} 
(2005), as Theorem 5 generalizes Hatfield and Milgrom (2005) by weakening the requirement on hospital preferences from the substitutes condition to the unilateral substitutes condition.

A mechanism is strategy-proof if, for any preference profile $P$, there is no doctor $d$ and preferences $P_{d}^{\prime}$ such that $d$ strictly prefers $y_{d}$ to $x_{d}$ according to $P_{d}$, where $x_{d}$ and $y_{d}$ are the doctor-optimal stable allocations for $d$ under $P$ and $\left(P_{d}^{\prime}, P_{-d}\right)$, respectively. A mechanism is group strategy-proof if, for any preference profile $P$, there is no group of doctors $D^{\prime} \subseteq D$ and a preference profile $P_{D^{\prime}}^{\prime}=\left(P_{d}^{\prime}\right)_{d \in D^{\prime}}$ such that every $d \in D^{\prime}$ strictly prefers $y_{d}$ to $x_{d}$ according to $P_{d}$, where $x_{d}$ and $y_{d}$ are the doctor-optimal stable allocations for $d$ under $P$ and $\left(P_{D^{\prime}}^{\prime}, P_{-D^{\prime}}\right)$, respectively. The doctor-optimal stable mechanism is a mechanism which, for any reported preference profile $P$, produces the doctor-optimal stable allocation under $P$.

Theorem 6. Suppose that preferences of every hospital satisfy unilateral substitutes and the law of aggregate demand. Then the doctor-optimal stable mechanism is group strategy-proof. In particular, the doctor-optimal stable mechanism is strategy-proof.

Proof. The proof is an adaptation of Hatfield and Milgrom (2005) and Hatfield and Kojima (2007a). First, proofs of Theorems 10 and 11 of Hatfield and Milgrom (2005) show that the doctor-optimal stable mechanism is strategy-proof if the doctor-optimal stable allocations exists and the conclusion of the rural hospital theorem (in the sense of Theorem 5) holds. The proof of Theorem 1 of Hatfield and Kojima (2007a) shows that the doctor-optimal stable mechanism is group strategy-proof if it is strategyproof. These observations and Theorems 4 and 5 in the current paper establish group strategy-proofness. Finally, since group strategy-proofness implies strategy-proofness in general, the doctor-optimal stable mechanism is strategy-proof.

Many incentive compatibility results in the literature are special cases of Theorem 6. Strategy-proofness was first obtained by Dubins and Freedman (1981) and Roth (1982) in one-to-one matching markets, and extended by Abdulkadiroğlu (2005) to more general preferences and then by Hatfield and Milgrom (2005) to the matching markets with contracts under the substitutes and the law of aggregate demand conditions. Group strategy-proofness was first shown by Dubins and Freedman (1981) in one-to-one matching. Martinez et al. (2004) obtained group strategy-proofness in many-to-one matching when hospitals' preferences satisfy substitutes and what they call $q$ separability, a condition stronger than the law of aggregate demand. Hatfield and Kojima (2007a) establishes group strategy-proofness under substitutes 
and the law of aggregate demand, generalizing previous results. All these results are special cases of Theorem 6 .

A possible extension of this result is the claim that no coalition can misreport preferences and make some of its members strictly better off without making any of its members strictly worse off. Unfortunately, such a result does not hold even in one-to-one matching markets (Dubins and Freedman, 1981). This fact suggests that, while outside the model, it may be possible for a coalition of doctors to manipulate the doctor-optimal stable mechanism if even small transfers are allowed among doctors. See section 4.3.1 of Roth and Sotomayor (1990) for exposition and discussion of this point.

Furthermore, Theorem 6 can not be further generalized to include coalitions including hospitals. In one-to-one matching markets, it is well-known that a single hospital can improve its outcome by not reporting truthfully under the doctor-optimal stable mechanism (Roth, 1982). Further, in manyto-one matching markets, even a single hospital can sometimes manipulate the hospital-optimal stable mechanism (Roth, 1985).

Our result can be used to derive another result that has been proven before in a number of special contexts. An immediate corollary of Theorem 6 is the following welfare result.

Corollary 2. Suppose that preferences of every hospital satisfy unilateral substitutes and the law of aggregate demand. Then there exists no individually rational allocation that every doctor strictly prefers to the doctor-optimal stable allocation.

Proof. Suppose by way of contradiction that there is an individually rational allocation $\left\{y_{d}\right\}_{d \in D}$ that each doctor strictly prefers to the doctoroptimal stable allocation. For each $d \in D$, let $P_{d}^{\prime}$ be preferences that declare $y_{d}$ as the unique acceptable contract. It is easy to show that $\left\{y_{d}\right\}_{d \in D}$ is the unique stable allocation when each $d$ declares $P_{d}^{\prime}$. This contradicts Theorem 6 , completing the proof.

This result is known as "weak Pareto optimality" in the literature and first presented by Roth (1982) for one-to-one matching. Martinez et al. (2004) obtain the result in many-to-one matching when hospitals' preferences satisfy substitutes and $q$-separability. Hatfield and Kojima (2007a) and Kojima (2007) establish this result under substitutes and the law of aggregate demand.

The results in this section are useful in understanding further properties of matching with couples. Theorem 4.3 of Klaus and Klijn (2005) gives an example in which different numbers of positions are filled in different stable matchings. It is easy to see that responsive preferences of a couple 
may violate the unilateral substitutes condition while satisfying the bilateral substitutes condition. Our results suggest that the reason for such instances is the lack of unilateral substitutes.

\section{Conclusion}

The matching problem with contracts subsumes a large class of problems, such as the matching model with fixed terms of contract, the job matching model with adjustable wages of Kelso and Crawford (1982) and the package auction model of Ausubel and Milgrom (2002). The current paper introduces two new conditions on preferences to obtain general results in the matching problem with contracts. Bilateral substitutes is sufficient for the existence of a stable allocation, and yet there does not necessarily exist a lattice of stable allocations or even a doctor-optimal stable allocation. We also introduce a new algorithm, the doctor-proposing deferred acceptance mechanism with renegotiation in order to find and prove the existence of this stable allocation. This is necessary as our more general model does not have the monotonic structure that earlier work in this area has been able to exploit. We also introduce a second condition, unilateral substitutes, which is sufficient for the existence of a doctor-optimal stable allocation; however, even with this condition the problem does not have the same monotonic structure as earlier models and hence we do not find the same structure on the set of stable allocations. The more traditional substitutes condition is sufficient for the lattice structure.

We conclude with some open questions. The set of stable allocations do not have a lattice structure with respect to doctor preferences even if the unilateral substitutes condition is imposed. Whether there is a lattice structure with respect to hospital preferences under unilateral substitutes is unknown. More generally, the underlying structure of the set of stable allocations, under bilateral or unilateral substitutes, is not fully understood.

\section{References}

Abdulkadiroğlu, Atila, "College Admission with Affirmative Action," International Journal of Game Theory, 2005, 33, 535-549.

- and Tayfun Sönmez, "School Choice: A Mechanism Design Approach," American Economic Review, 2003, 93, 729-747. 
_, Parag A. Pathak, Alvin E. Roth, and Tayfun Sönmez, "The Boston Public School Match," American Economic Review Papers and Proceedings, 2005, 95, 368-372.

_ , _, and _, "The New York City High School Match," American Economic Review Papers and Proceedings, 2005, 95, 364-367.

Adachi, Hiroyuki, "On a characterization of stable matchings," Economics Letters, 2000, 68, 43-49.

Alkan, Ahmet, "A class of multipartner matching markets with a strong lattice structure," Economic Theory, 2002, 19 (4), 737-746.

- and David Gale, "Stable Schedule Matching Under Revealed Preferences," Journal of Economic Theory, 2003, 84, 73-94.

Ausubel, Lawrence M. and Paul R. Milgrom, "Ascending Auctions with Package Bidding," Frontiers of Theoretical Economics, 2002, 1, 1.

Dubins, L. E. and D. A. Freedman, "Machiavelli and the Gale-Shapley algorithm," American Mathematical Monthly, 1981, 88, 485-494.

Echenique, Federico and Jorge Oviedo, "Core Many-to-One Matchings by Fixed Point Methods," Journal of Economic Theory, 2004, 115, 358376.

_ and _ , "A theory of stability in many-to-many matching," Theoretical Economics, 2006, 1, 233-273.

Fleiner, Tamás, "A Fixed-Point Approach to Stable Matchings and Some Applications," Mathematics of Operations Research, 2003, 28, 103-126.

Gale, David and Lloyd S. Shapley, "College Admissions and the Stability of Marriage," American Mathematical Monthly, 1962, 69, 9-15.

Hatfield, John and Fuhito Kojima, "Group Incentive Compatibility for Matching with Contracts," 2007. mimeo.

_ and _, "Matching with Contracts: Corrigendum," 2007. American Economic Review, forthcoming.

- and Paul R. Milgrom, "Matching with Contracts," American Economic Review, 2005, 95, 913-935.

Kelso, Alexander and Vincent P. Crawford, "Job matching, coalition formation, and gross substitutes," Econometrica, 1982, 50, 1483-1504. 
Klaus, Bettina and Flip Klijn, "Stable matchings and preferences of couples," Journal of Economic Theory, 2005, 121, 75-106.

_, _, and Toshifumi Nakamura, "Corrigendum: Stable Matchings and Preferences of Couples," 2006. forthcoming, Journal of Economic Theory.

Kojima, Fuhito, "The Law of Aggregate Demand and Welfare in the TwoSided Matching Market," Economics Letters, 2007, forthcoming.

- and Parag A. Pathak, "Incentives and stability in large two-sided matching markets," 2007. mimeo.

Konishi, Hideo and Utku Ünver, "Credible group stability in many-tomany matching problems," Journal of Economic Theory, 2006, 129 (1), $57-80$.

Martinez, Ruth, Jordi Masso, Alejandro Neme, and Jorge Oviedo, "Single agents and the set of many-to-one stable matchings," Journal of Economic Theory, 2000, 91, 91-105.

_ , _ , , and _, "On group strategy-proof mechanisms for a many-toone matching model," International Journal of Game Theory, 2004, 33, $115-128$.

Ostrovsky, Michael, "Stability in Supply Chain Networks," American Economic Review, 2007, forthcoming.

Roth, Alvin E., "The Economics of Matching: Stability and Incentives," Mathematics of Operations Research, 1982, 7, 617-628.

_, "The Evolution of the Labor Market for Medical Interns and Residents: A Case Study in Game Theory," Journal of Political Economy, 1984, 92, 991-1016.

_ , "Stability and Polarization of Interests in Job Matching," Econometrica, 1984, 52 (1), 47-58.

_ , "Conflict and Coincidence of Interest in Job Matching: Some New Results and Open Questions," Mathematics of Operations Research, 1985, 10, 379389.

_ , "On the allocation of residents to rural hospitals: a general property of two-sided matching markets," Econometrica, 1986, 54, 425-427. 
- and Elliott Peranson, "The Redesign of the Matching Market for American Physicians: Some Engineering Aspects of Economic Design," American Economic Review, 1999, 89, 748-780.

- and John Vande Vate, "Incentives in two-sided matching with random stable mechanisms," Economic Theory, 1991, 1, 31-44.

- and Marilda A. O. Sotomayor, Two-sided matching: a study in gametheoretic modeling and analysis, Cambridge: Econometric Society monographs, 1990.

- and Uriel Rothblum, "Truncation Strategies in Matching Markets: In Search of Advice for Participants," Econometrica, 1999, 67, 21-43. 